Original Paper http://ajol.info/index.php/ijbes http://indexmedicus.afro.who.int

\title{
Perceptions paysannes de la dégradation des ressources naturelles des bas-fonds en zone soudano-sahélienne: cas du sous bassin versant du Nakanbé-Dem au Burkina Faso
}

\author{
Adama ILBOUDO ${ }^{*}$, Soungalo SOULAMA ${ }^{2}$, Edmond HIEN $^{1}$ et Prosper ZOMBRE ${ }^{1}$ \\ ${ }^{1}$ Laboratoire Sols, Matériaux et Environnement, Université Ouaga I Pr Joseph KI-ZERBO, 03 BP 7021 \\ Ouagadougou 03, Burkina Faso. \\ ${ }^{2}$ Département Productions Forestières, INERA, CNRST 03 BP 4076 Ouagadougou 03, Burkina Faso. \\ *Auteur correspondant ; E-mail : ilboudama@yahoo.fr ; Tel +22670 711112
}

\section{RESUME}

Le Sahel burkinabé est caractérisé par une forte dégradation des ressources naturelles. Cette régression est imputable à des facteurs naturels et anthropiques. La présente étude concerne les bas-fonds du sous bassin versant du Nakanbé-Dem situé dans la limite australe du Sahel. Dans cette zone dominée par l'agriculture pluviale et l'élevage extensif, les zones humides revêtent une importance capitale. Face à leur dégradation continue, la participation des communautés est nécessaire pour une gestion durable des bas-fonds. Cette étude analyse les indicateurs endogènes de la dégradation des bas-fonds de la zone soudano-sahélienne. Des données d'enquêtes semi-structurées ont été collectées auprès de 325 exploitants de six bas-fonds. Des statistiques descriptives et une analyse factorielle par correspondance (AFC) ont été appliquées. Les résultats montrent que les paysans perçoivent clairement la dégradation des bas-fonds. Les paysans se servent d'éléments météorologiques, floristiques et physiques pour caractériser l'état de dégradation des bas-fonds. La disparition de plusieurs espèces et l'apparition d'espèces ubiquistes leur permettent d'évaluer la dégradation des terres des bas-fonds. Ces indicateurs endogènes varient en fonction du sexe et du niveau d'instruction. L'étude a souligné l'importance des connaissances endogènes dans l'analyse du phénomène de dégradation des bas-fonds. Elle suggère la nécessité d'en tenir compte désormais dans la mise en œuvre des techniques de restauration des basfonds dégradés.

(C) 2020 International Formulae Group. All rights reserved.

Mots clés : Indicateurs endogènes ; dégradation des bas-fonds ; perceptions ; bassin versant, Burkina Faso.

\section{Farmers' Perceptions of lowland's natural resources degradation in Sudano Sahelian area: case of Nakanbe-Dem Sub-Watershed in Burkina Faso}

\begin{abstract}
The Burkina Faso Sahel is characterized by a great degradation of natural resources. This regression is due to natural and anthropogenic factors. This study concerns the lowlands of the Nakanbé-Dem sub-watershed, located in the northern limit of the Sahel. In this area dominated by rain farming and extensive livestock, wetlands are very important. In this context of continuous lowlands degradation, the participation of communities is necessary for a sustainable management. This study analyses the local indicators of lowlands' degradation in
\end{abstract}


Sudano-Sahelian zone. Data from semi-structured surveys were collected from 325 farmers in six lowlands of Nakanbé-Dem sub-watershed. Descriptive statistics and correspondence factor analysis (CFA) were applied. The results show that farmers clearly perceive the degradation of the lowlands. Farmers use meteorological, floristic and physical elements to characterize the state of lowlands degradation. The loss of several species and the appearance of ubiquitous species allow them to assess the degradation of the lowlands. These local indicators vary according to sex and education level. Farmers also apprehend degraded lowlands flora reshaping and identify taxa indicative of lowland ecosystems health. The study highlighted the importance of the endogenous knowledge in the analysis of lowlands' degradation. It suggests the need to take into account local indicators in the implementation of lowlands' restoration techniques.

(C) 2020 International Formulae Group. All rights reserved.

Keywords: endogenous indicators, lowlands degradation, perception, Watershed, Burkina Faso.

\section{INTRODUCTION}

Les bas-fonds en région intertropicale représentent une zone du paysage à fond plat ou concave. Ils sont situés à la tête du réseau hydrographique, sans cours d'eau ou avec un lit mineur peu marqué, et constituent des axes de drainage élémentaire (Hounkpetin, 2003). Ils se distinguent des plaines alluviales qui leur font suite en aval, et ont une hydrologie et une morphologie différentes par le développement $\mathrm{du}$ bourrelet de berge, le remaniement pédologique, l'inondation par l'aval (Raunet, 1985).

Dans les pays sahéliens, les bas-fonds sont des zones humides qui connaissent une pression anthropique de plus en plus accentuée avec les variabilités climatiques (Souberou et al., 2017). Les représentations des communautés agricoles de ces unités bioproductives constituent un objet d'étude pertinent dans un contexte de changements globaux. Ainsi, les pratiques d'adaptation développées par les producteurs en réponse aux conséquences négatives des changements climatiques dépendent de la perception et des connaissances endogènes qu'ils ont de ces changements.

Au Burkina Faso, la succession des sécheresses des années 1970 et 1980 qu'a connue l'Afrique sub-Sahélienne s'est traduite par la dégradation des écosystèmes et une diminution du potentiel productif. La région de Kaya a été particulièrement touchée. Les basfonds, jadis considérés comme des terres incultes sur le plan agricole, étaient utilisés, de ce fait par les éleveurs et dans certains cas comme lieux de cultes. Aujourd'hui, ces zones humides sont devenues des terres agricoles convoitées par les paysans (Yacouba et al., 2002).

La pression foncière sur les bas-fonds est favorisée par les potentialités qu'offrent ces zones humides, espaces multi-usages, et considérées comme des sols à hauts rendements agricoles. La mise en valeur des bas-fonds permet aux producteurs de tirer profit des potentiels pédologiques et hydrologiques disponibles et des opportunités socio- économiques (Souberou et al., 2018).

Sous la conjugaison des péjorations climatiques et des activités humaines, les basfonds non encore aménagés de la zone soudano-sahélienne, connaissent une évolution environnementale marquée principalement par la dégradation des versants, l'assèchement des cours d'eau, le recul de la nappe phréatique, la disparition et la dégradation du couvert végétal ainsi que la régression de la phytodiversité (Bambara et al., 2013). La végétation et la flore des bas-fonds, caractéristiques des milieux fréquemment inondés, subissent également un changement (Soulama et al., 2015). Les indicateurs de changement de leur environnement sont perçus par les paysans. La perception locale est une approche indispensable pour l'étude de la dégradation des écosystèmes. En outre, elle garantit l'acceptation locale des perspectives et stratégies d'aménagements. L'objectif de cette étude est d'analyser les indicateurs endogènes de dégradation des bas-fonds du sous bassin versant du Nakanbé-Dem, exploités par des hommes et des femmes dont certains bénéficient de la part de l'Etat et des 
Organisations Non Gouvernementales d'appuis et de renforcement des capacités pour la gestion durable des bas-fonds. L'hypothèse de travail est que les indicateurs endogènes de dégradation des bas-fonds varient selon le sexe et le niveau d'instruction.

\section{MATERIEL ET METHODES Sites d'étude}

L'étude a été conduite sur le sous bassin versant du Nakanbé-Dem situé dans la Région du Centre-Nord du Burkina Faso (Afrique de l'Ouest). Le sous bassin versant du NakanbéDem, d'une superficie de 165029 hectares (ha), est reparti entre les longitudes $1^{\circ} 26^{\prime} 30^{\prime \prime}$ $\mathrm{W}$ et $0^{\circ} 57^{\prime} 43^{\prime \prime} \mathrm{W}$ et les latitudes $13^{\circ} 58^{\prime} 58^{\prime \prime} \mathrm{N}$ et $13^{\circ} 31^{\prime} 48 \mathrm{~N}$. Il se trouve dans le secteur phytogéographique nord-soudanien (Fontès et Guinko, 1995) et connait un régime pluviométrique unimodal.

La seule saison pluvieuse s'étale de juin à octobre. Les précipitations moyennes annuelles oscillent entre 600 et $700 \mathrm{~mm}$. Les températures moyennes annuelles varient entre 20 à $28^{\circ} \mathrm{C}$ pendant la saison humide et sont par contre très élevées en saison sèche, 35 à $40^{\circ} \mathrm{C}$, favorisant l'assèchement des points d'eau par l'évaporation (Da et al., 2008). Sur le plan géologique, la zone d'étude comporte deux types de formations, l'anté-birrimien composé des formations granitiques et le Birimien composé essentiellement de roches volcanosédimentaires. Les collines birimiennes constituent l'ossature du modelé de la zone d'étude. Sur ces unités géomorphologiques, se développent cinq classes de sols à savoir, les sols minéraux bruts, les sols peu évolués, les sols brunifiés, les sols à sesquioxydes de fer et/ou de manganèse et les sols hydromorphes (CPCS, 1967). Le paysage géomorphologique s'organise depuis les reliefs résiduels jusqu'aux bas-fonds qui constituent des zones préférentielles d'accumulation des eaux de ruissellement descendant à partir des versants des collines avoisinantes. Les bas-fonds sont aussi des zones de forte recharge des nappes souterraines et c'est le long de ces bas-fonds que les retenues d'eau et autres ouvrages d'aménagement hydro-agricole sont construits. Jadis considérés comme des terres incultes sur le plan agricole, réservés aux pâturages et dans certains cas aux lieux de cultes, les bas-fonds sont aujourd'hui des terres agricoles convoitées par les paysans du fait de leur richesse en bases échangeables et en matières organiques. Les formations végétales dégradées sont principalement composées de savanes-parcs dans lesquelles les forêts galeries ont quasiment disparu (Da et al., 2008). La pression anthropique et la péjoration climatique ont contribué à la forte réduction du couvert végétal entrainant une extension des zones des sols nus. Dans le secteur subsahélien, correspondant à notre zone d'étude, la végétation des zones humides telles que les bas-fonds est caractérisée par Mitragyna inermis, Combretum micranthum, Feretia apodanthera, Anogeissus leiocarpa, Crateva adansonii, Flueggea virosa, Diospiros mespiliformis, Acacia ataxancatha, Ximenia americana, Gardenia ternifolia, Dichrostachys cinerea, Tapinanthus globiferus, Acacia erythrocalyx, Grewia flavescens, Terminalia glauscessens (Sambare et al., 2011).

La population est constituée de $86,27 \%$ d'ethnie mossi et de $9,7 \%$ de peulh. Elle est caractérisée par une croissance démographique de $2,6 \%$ en moyenne. Le taux d'alphabétisation des individus de 15 ans et plus était de 24,9\% en 2014 (INSD, 2009). L'activité agricole occupe plus de $80 \%$ de la population active. L'agriculture, essentiellement pluviale, est de type extensif et peu mécanisée. Elle est pratiquée cumulativement avec l'élevage qui constitue la deuxième grande activité (INSD, 2009). La population du sous bassin du Nakanbé-Dem, toujours en quête des terres fertiles, intervient activement dans la valorisation des bas-fonds constituant un palliatif aux variabilités climatiques. Notre étude a porté sur six bas-fonds du sous bassin du Nakanbé-Dem qui sont localisés dans les provinces de Sanmatenga et de Bam (Figure 1).

\section{Collecte des données}

Sur un total de 650 exploitants, un échantillon de 325 enquêtés a été constitué sur la base d'un échantillonnage stratifié et aléatoire, soit $50 \%$ de la population des exploitants des bas-fonds. Les strates sont basées sur le sexe et le niveau d'éducation. L'unité statistique est une femme ou un homme 
ressortissant d'un des six villages riverains des bas-fonds, âgé d'au moins 18 ans et ayant exploité le bas-fond durant les cinq dernières années. Les données ont été collectées à partir d'un questionnaire structuré. Les interviewés ont été sollicités pour répondre aux questions relatives aux indicateurs de dégradation des bas-fonds, aux taxons biologiques qui caractérisent les bas-fonds dégradés ainsi qu'aux causes de la dégradation et les menaces affectant ces bas-fonds.

Les interviews individuelles ont été réalisées en langue locale mooré. Cependant, pour l'identification des espèces indicatrices de l'état de santé des bas-fonds, un focus group a eu lieu avec des hommes et des femmes d'âge avancé. Des échantillons d'herbiers ont été constitués pour l'identification et la comparaison avec les spécimens de l'herbier du laboratoire de biologie et écologie végétales de l'Université Joseph KI-ZERBO. Les noms vernaculaires ont été vérifiés à l'aide des flores notamment de Thiombiano et al. (2012) et de
Nacoulma-Ouédraogo

(1996).

$\mathrm{La}$ nomenclature adoptée est celle du catalogue des plantes vasculaires du Burkina Faso (Lebrun et Stork , 1991).

\section{Analyse des données}

Les données ont été analysées à l'aide de statistiques descriptives (fréquences et moyennes) pour établir la cause de la dégradation des ressources naturelles des basfonds, l'impact des péjorations climatiques sur ces ressources et la dynamique de la biodiversité des bas-fonds. Par ailleurs, une analyse factorielle par correspondance (AFC) a été utilisée pour analyser les facteurs de la dégradation des bas-fonds selon le sexe et le niveau d'instruction des paysans à l'aide du logiciel R. Selon certains auteurs, les caractéristiques socio-économiques du ménage telles que l'âge, le sexe, le niveau d'éducation et la taille de l'exploitation influencent son adaptation et l'adoption de nouvelles méthodes (Nana et Thiombiano, 2018).

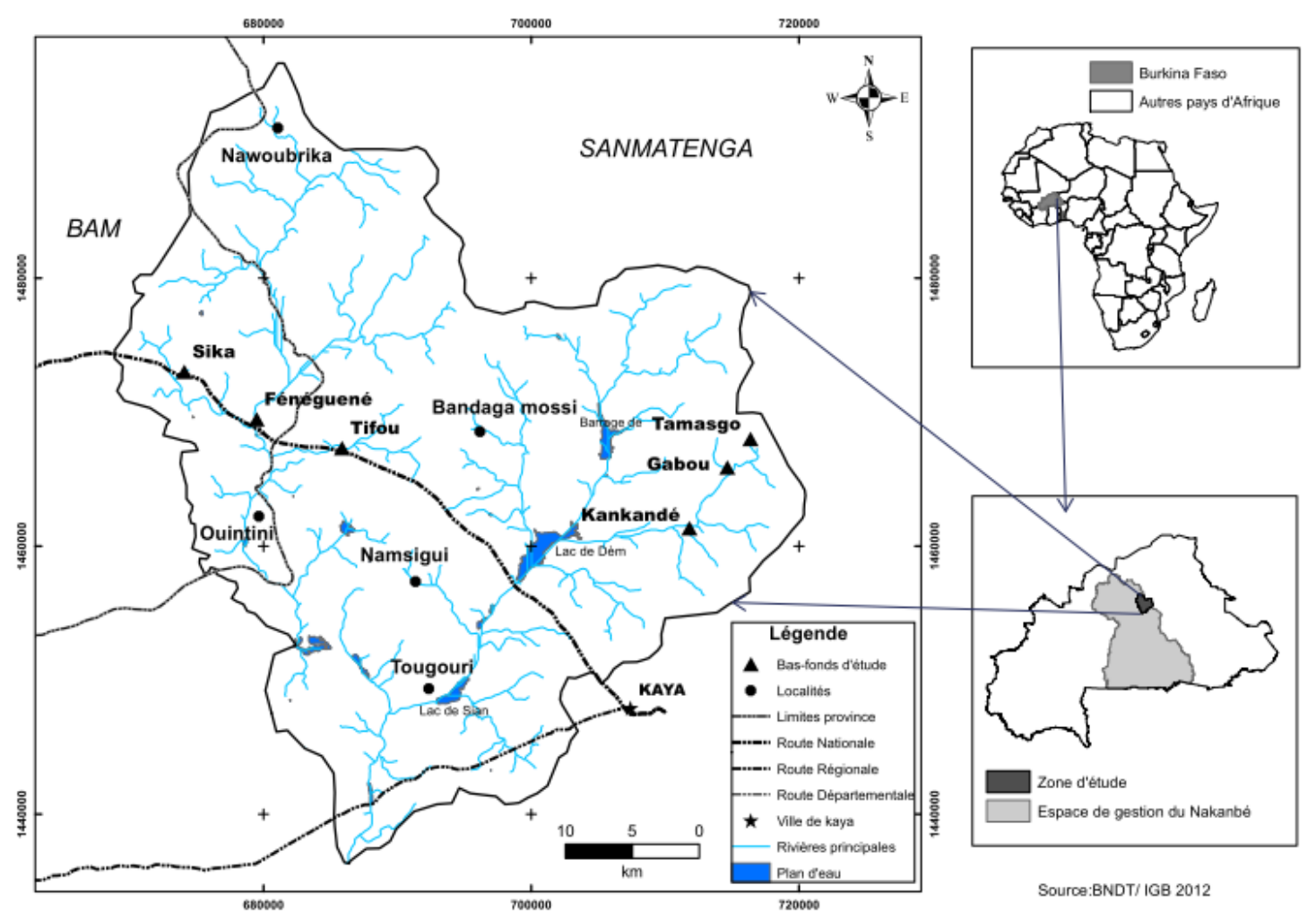

Figure 1: Localisation des sites d'étude. 


\section{RESULTATS}

\section{Caractéristiques socio-démographiques}

L'échantillon des exploitants des basfonds (Tableau 1) est dominé par les hommes. Les exploitants non alphabétisés sont deux fois plus nombreux que les alphabétisés. Les exploitants enquêtés pratiquent principalement l'agriculture pluviale et accessoirement l'élevage, le maraîchage, l'exploitation des plantes, la maçonnerie et l'artisanat.

\section{Causes de la dégradation des bas-fonds selon les enquêtés}

La Figure 2 donne l'analyse de la perception des exploitants des facteurs de dégradation des bas-fonds en fonction du sexe et du niveau d'instruction. Les deux premiers axes expliquent $95,71 \%$ de la dimension expliquée. L'axe 2 montre un gradient du niveau d'instruction, il sépare les alphabétisés des non alphabétisés. Quant à l'axe1, il sépare les femmes des hommes.

L'analyse des variables ayant fortement contribué à l'inertie des axes montre que, les hommes alphabétisés perçoivent le labour mécanique et les inondations comme étant les principaux facteurs de dégradation des agrosystèmes des bas-fonds tandis que, les hommes non alphabétisés indexent les vents violents et le surpâturage comme principaux facteurs de dégradation. Quant aux femmes alphabétisées, les poches de sécheresse fréquentes, les feux de brousse et la coupe du bois constituent les principaux facteurs de dégradation des bas-fonds. Le raccourcissement de la durée de la saison est perçu comme étant le principal facteur de dégradation de ces bas-fonds par les femmes non alphabétisées.

\section{Perception des signes de dégradation des bas-fonds}

La Figure 3 donne l'analyse de la perception des exploitants des signes de dégradation des bas-fonds en fonction du sexe et du niveau d'instruction. Les deux premiers axes expliquent $99,20 \%$ de la dimension expliquée.

Aussi bien les femmes alphabétisées que les femmes non alphabétisées perçoivent le séjour moins long de l'eau, la dégradation du sol et le ravinement des bas-fonds comme étant les indicateurs physiques de dégradation de ces milieux humides.

Quant aux hommes alphabétisés, ils perçoivent les plaquages de sable et de gravillons ainsi que l'élargissement de la zone centrale des bas-fonds comme les principaux signes de dégradation, tandis que les hommes non alphabétisés perçoivent la diminution du couvert végétal et du nombre d'arbres autour des bas-fonds comme principaux indicateurs de leur dégradation.

\section{Perception de l'état des ressources phytobiologiques des bas-fonds dégradés}

Les données agrégées sur les citations des espèces végétales indicatrices de l'état des bas-fonds, ont permis de retenir celles ayant fait le plus de consensus chez les exploitants des bas-fonds. Ainsi, il apparaît que les exploitants des bas-fonds du Nakanbé-Dem perçoivent l'évolution phytobiologique de leurs bas-fonds. Ils reconnaissent les espèces qui prolifèrent actuellement dans les bas-fonds dégradés tout comme les espèces ayant disparu de ces sites (Tableau 2).

Tableau 1 : Caractéristiques socio-démographiques des exploitants des bas-fonds.

\begin{tabular}{lcc}
\hline Sexe & Effectif & Pourcentage (\%) \\
\hline Homme & 194 & 59,69 \\
Femme & 131 & 40,30 \\
Niveau d'instruction & & \\
Non alphabétisés & 202 & 62,15 \\
Alphabétisés & 123 & 37,80 \\
\hline
\end{tabular}




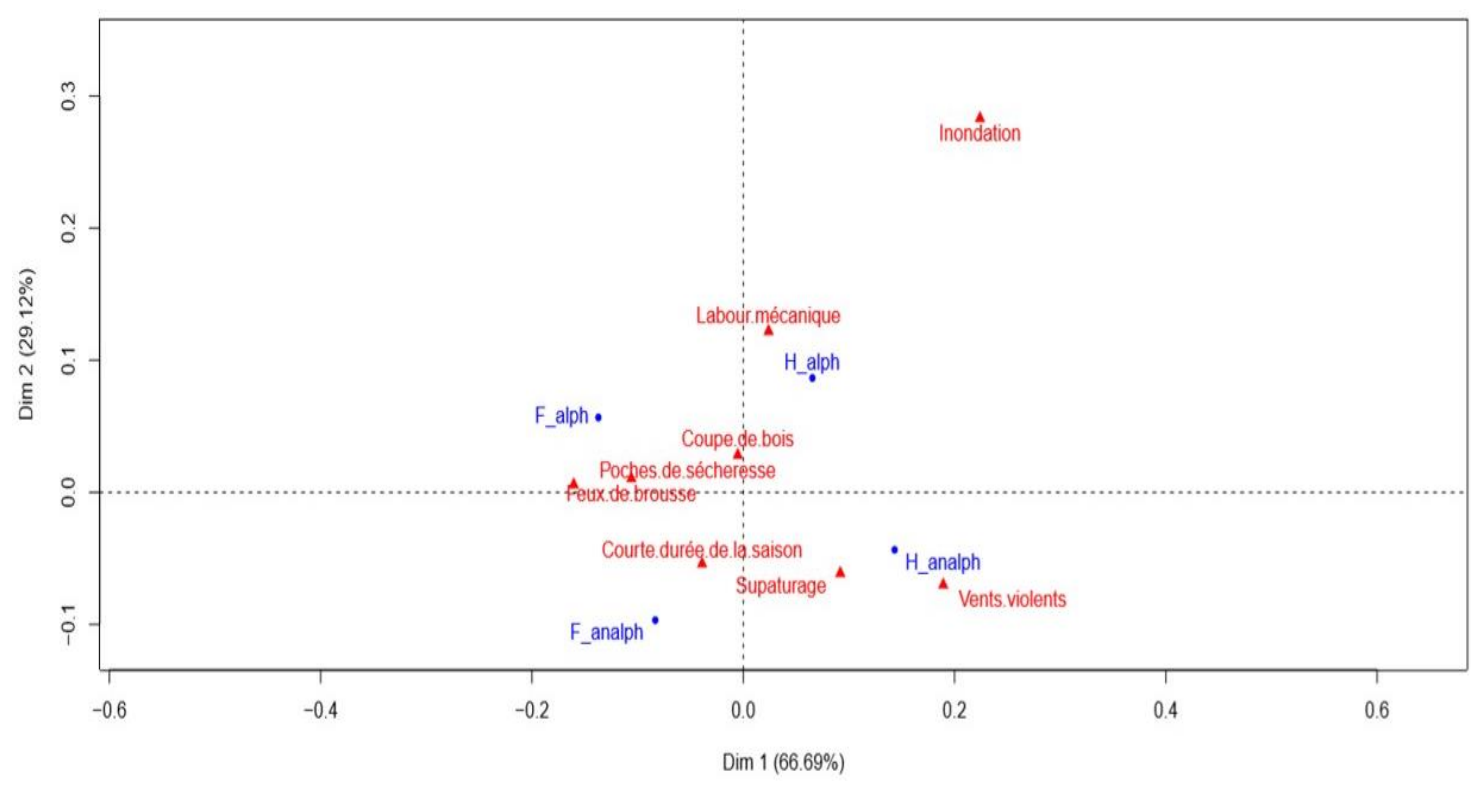

F_alph : Femmes alphabétisées, F_analph : Femmes non alphabétisées, H_alph : Hommes alphabétisés H_analph : Hommes non alphabétisés.

Figure 2: Analyse des facteurs de dégradation des bas-fonds en fonction du sexe et du niveau d'alphabétisation.

Tableau 2: Etat des ressources phytobiologiques des bas-fonds.

\begin{tabular}{|c|c|c|c|c|}
\hline \multicolumn{5}{|c|}{ Arbres en prolifération dans les bas-fonds dégradés } \\
\hline Nom local & Espèce & Famille & $\mathbf{T P}$ & TB \\
\hline Kiègla & Balanites aegyptiaca (L.) Del. & Zygophyllaceae & $\mathrm{SZ}$ & $\mathrm{mph}$ \\
\hline Pennga & Acacia nilotica (L.) Willd. ex Del & Fabaceae & SZ & $\mathrm{mph}$ \\
\hline Mugunuga & Ziziphus mauritiana Lam. & Rhamnaceae & Pal & $\mathrm{mph}$ \\
\hline Randga & Combretum micranthum G. Don & Combretaceae & $S$ & $\mathrm{mph}$ \\
\hline Banguemdé & Piliostigma reticulatum (DC.) Hochst. & Fabaceae & $\mathrm{SZ}$ & $\mathrm{mph}$ \\
\hline \multicolumn{5}{|c|}{ Arbres disparus dans les bas-fonds dégradés } \\
\hline Nom local & Espèce & Famille & $\mathbf{T P}$ & TB \\
\hline Gonpagnatga & Senegalia senegal (L.) Britton) & Fabaceae & $\mathrm{SZ}$ & $\mathrm{mph}$ \\
\hline Kombrayinogo & Boswellia dalzielii Hutch. & Burseraceae & $\mathrm{S}$ & $\mathrm{mph}$ \\
\hline bargôdga & Annona senegalensis Pers. & Annonaceae & S & $\mathrm{nph}$ \\
\hline siiga & Anogeissus leiocarpa (DC.) Guill. \& Perr. & Combretaceae & $S$ & $\mathrm{mph}$ \\
\hline Vouaga & Bombax costatum Pellegr. et Vuillet. & Malvaceae & $S$ & $\mathrm{mph}$ \\
\hline Yilga & Mitragyna inermis (Willd.) Kuntze & Rubiaceae & $\mathrm{SZ}$ & $\mathrm{mph}$ \\
\hline Lènga & Ximenia americana $L$. & Olacaceae & Pan & $\mathrm{nph}$ \\
\hline
\end{tabular}


Faranguemtoula

Capparis sepiaria L. (C. corymbosa Lam).

Kaanka

Diospyros mespiliformis Hoschst.ex A. DC.

Wilinwiga

Guiera senegalensis J. G. Gmel.

Kuka Khaya senegalensis (Desr.) A. Juss.

Nayüponka

Pterocarpus erinaceus Poir.

Capparacae

S Lnph

Kalgmetoèga

Crateva adansonii DC.

Ebenaceae

Pal mph

Combretaceae SZ mph

Meliaceae $\quad S \quad \mathrm{mph}$

Fabaceae $\quad \mathrm{SZ} \quad \mathrm{mPh}$

Capparaceae Pal mph

Herbacées en prolifération dans les bas-fonds dégradés

\begin{tabular}{lllll}
\hline Nom local & \multicolumn{1}{c}{ Espèce } & Famille & TP & TB \\
\hline Pintme-pin & Digitaria horizontalis Willd. & Poaceae & Pan & Th \\
Kimbgo & Pennisetum pedicellatum Trin. & Poaceae & Pal & Th \\
Roumroumbi & Hyptis spicigera Lam. & Lamiaceae & Pan & Th \\
Sangodo & Senna obtusifolia (L.) Irwin \& Barneby & Fabaceae & Pan & Th \\
Wango & Striga hermonthica (Delile) Benth. & Orobanchaceae & AT & He \\
Monpoaka & Andropogon gayanus Kunth & Poaceae & S & He \\
Naasar-gonsé & Acanthospermum hispidum DC. & Asteraceae & Pan Th \\
Boango-yando & Commelina benghalensis L. & Commelinaceae & Pan Ch & PA \\
Kansaga & Sporobolus pyramidalis P.Beauv. & Poaceae & AA \\
\hline
\end{tabular}

Herbacées disparues dans les bas-fonds dégradés

\begin{tabular}{|c|c|c|c|c|}
\hline Nom local & Espèce & Famille & $\mathbf{T P}$ & TB \\
\hline Gnantré & Andropogon pseudapricus Stapf & Poaceae & $\mathrm{AA}$ & $\mathrm{Th}$ \\
\hline Kaloêga & Schizachyrium sanguineum (Retz.) Alston & Poaceae & Pan & $\mathrm{He}$ \\
\hline Koulmoogo & Oryza longistaminata A. Chev. \& Roehr. & Poaceae & $\mathrm{AM}$ & $\mathrm{He}$ \\
\hline Gouila & Nymphea lotus $L$. & Nympheaceae & Pan & $\mathrm{Ge}$ \\
\hline Goadba & Stylochaeton hypogaeus Lepr. & Araceae & $\mathrm{S}$ & $\mathrm{Ge}$ \\
\hline Doudouma & Vetiveria nigritana (Benth.) Stapf & Poaceae & $\mathrm{SZ}$ & $\mathrm{He}$ \\
\hline Remsa & $\begin{array}{l}\text { Alysicarpus } \\
\text { (Schum.\&Thonn)J.L }\end{array}$ & Fabaceae & Pal & Th \\
\hline Kiwaré & Cymbopogon giganteus Chiov. & Poaceae & $\mathrm{AT}$ & $\mathrm{He}$ \\
\hline Kalgên yâaga & $\begin{array}{l}\text { Andropogon tectorum Schumach.\& } \\
\text { Thonn. }\end{array}$ & Poaceae & $\mathrm{S}$ & $\mathrm{He}$ \\
\hline Pouloumdi & Imperata cylindrica (L.) P. Beauv. & Poaceae & Pan & $\mathrm{Ge}$ \\
\hline Kansaga & Sporobolus pyramidalis P. Beav. & Poaceae & AA & $\mathrm{He}$ \\
\hline
\end{tabular}

TP : Type phytogéographique, AA : Espèces à distribution afro-américaine, AM : Afro-malgaches, AT : Afrique tropicale , Pal : Paléotropicales , Pan: Pantropicales, PRA : Pluri-régionale africaines , S : Soudaniennes , SZ : Soudano-zambéziennes , TB : Type biologique, Ch : Chaméphyte, Chsl : Chaméphyte sous ligneux, Ge : Géophyte, He : Hémicryptophyte, Lnph : Liane Nanophanérophyte, Lth : Liane thérophyte , mph: Mesophanérophytes, nph: Nanophanérophytes, Ph : Phanérophytes, Th: Thérophytes. 


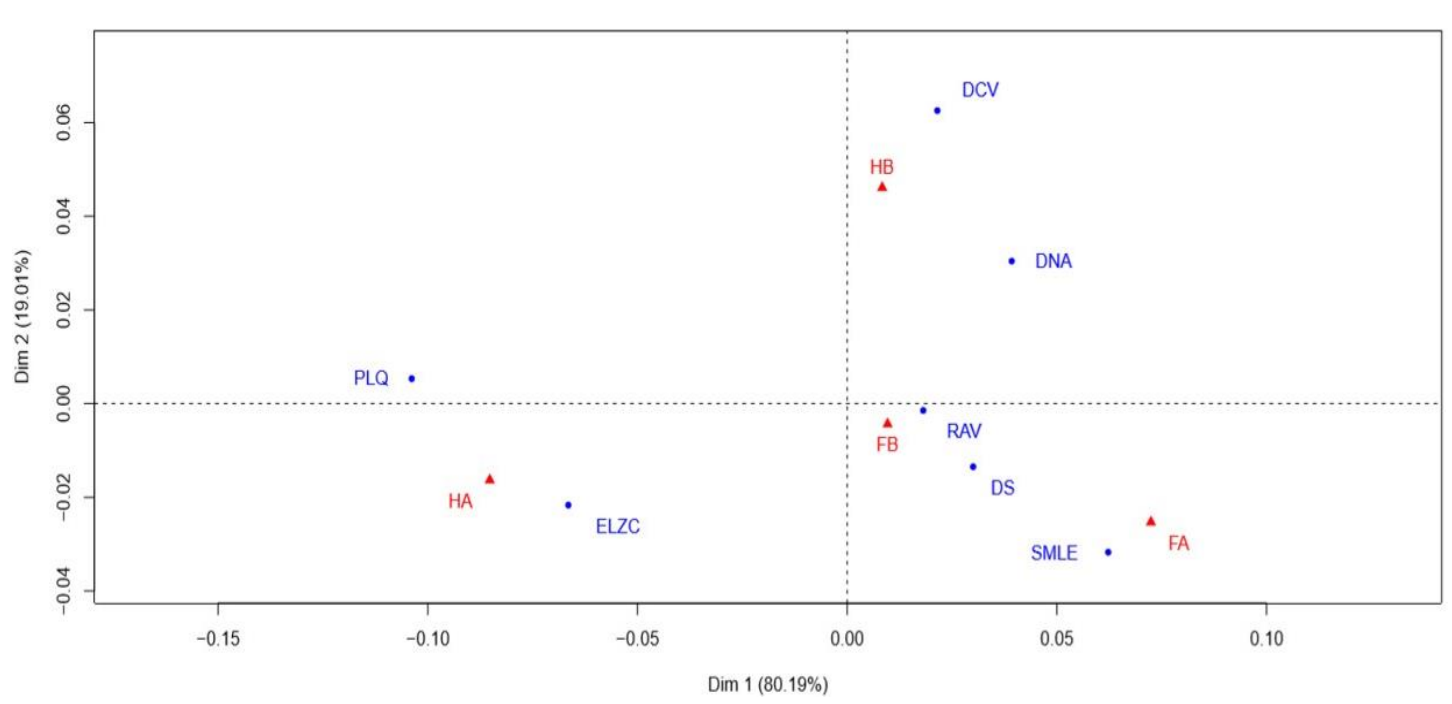

FA : Femmes alphabétisées, FB : Femmes non alphabétisées, HA : Hommes alphabétisés, HB : hommes non alphabétisés, DCV : Diminution du couvert végétal, DNA : Diminution du nombre d'arbres, DS : Dégradation du sol, ELZC : Élargissement de la zone centrale, PLQ : Placage sableux ou épandage de graviers, RAV : Ravinement, SMLE : Séjour moins long de l'eau.

Figure 3 : Analyse factorielle de la perception des menaces selon le sexe et le niveau d'instruction.

\section{DISCUSSION}

\section{Perception des facteurs de dégradation des bas-fonds}

Les bas-fonds du sous bassin versant du Nakanbé-Dem sont de plus en plus exploités pour l'agriculture. Les hommes sont plus nombreux à exploiter ces bas-fonds par rapport aux femmes. Cette tendance est générale en zone soudano-sahélienne où les bas-fonds connaissent, ces dernières décennies, une exploitation agricole intense en raison de la fertilité de leurs sols et de leur caractère hydromorphe (Souberou et al., 2017; Souberou et al., 2018). L'exploitation des bas-fonds constitue l'une des stratégies d'adaptation des agriculteurs au changement climatique. Dès lors, l'accès des femmes aux bas-fonds est de plus en plus réduit au détriment des hommes. Des recherches ont montré que la même tendance d'accès s'observe lorsque les basfonds sont aménagés pour des fins d'exploitation agricole (Huat et al., 2019).

Les communautés rurales du sous bassin versant du Nakanbé-Dem perçoivent bien la dégradation des bas-fonds ainsi que les facteurs qui en sont à l'origine. Cette perception varie selon le sexe et le niveau d'éducation. Cela ressort dans l'analyse factorielle de correspondance où l'axe 2 montre un gradient du niveau d'instruction tandis que l'axe 1 sépare les femmes des hommes.

Les agriculteurs du sous bassin versant du Nakanbé-Dem ont conscience que certaines de leurs pratiques dégradent les bas-fonds. Les hommes alphabétisés indexent le labour mécanique comme facteur anthropique de dégradation des bas-fonds. Le labour mécanique accentue l'érosion hydrique et le transport des sédiments pouvant combler les lits des bas-fonds. Dans un contexte où les sols sont peu profonds, le labour attelé dégrade les sols de bas-fonds. L'éducation et la formation reçues par certains exploitants des bas-fonds leur permettent de percevoir l'impact négatif des technologies comme le labour attelé sur les sols de bas-fonds. Ce résultat corrobore Sofoluwe et al. (2011) pour qui l'éducation et la formation accroissent la capacité des agriculteurs à utiliser des informations variées et influencent l'adoption des innovations technologiques. Il est donc nécessaire d'améliorer l'éducation formelle et informelle dans les communautés rurales pour une exploitation durable des bas-fonds. 
Le surpâturage est perçu par les hommes non alphabétisés comme étant le principal facteur anthropique qui menace les bas-fonds sans doute à cause de la forte densité du cheptel dans la zone du Nakanbé-Dem. Da et al. (2008) relèvent que les pratiques agropastorales dans la région du centre-Nord ont eu pour conséquences la dégradation des sols et une baisse notable de la fertilité y compris dans les zones humides. La densité du cheptel est plus élevée que la capacité de charge des basfonds. Le surpâturage dégrade la végétation des bas-fonds, compacte les sols qui deviennent susceptibles à l'encroûtement (Malam, 2007 ; Diallo et al., 2011; Enete et Onyekuru, 2011).

Enfin, les femmes non alphabétisées perçoivent la coupe du bois et les feux de brousse comme principaux facteurs anthropiques de dégradation des bas-fonds. Dans cette zone, la dynamique d'occupation des bas-fonds est également la conséquence de la pratique de brûlis associée à l'abattage des arbres pour la fabrication de charbon de bois et l'obtention du bois de chauffe (Oloukoi et al., 2006). Ces actions exposent les bas-fonds à divers problèmes dont la perte de leur potentialité, la dégradation des versants et des plateaux environnants (Diallo et al., 2011; Enete et Onyekuru, 2011). Ces mauvaises pratiques sont perçues par les femmes non alphabétisées en particulier. Cela peut être dû au fait qu'en milieu rural, la place et le rôle de la femme dans la cuisine, la prédisposent à s'apercevoir de la dégradation de la ressource «bois de feux » des écosystèmes des bas-fonds (Jennifer et al., 2004). De plus, face à l'instabilité récurrente des productions agricoles, les populations rurales, notamment les femmes développent des stratégies de survie dont une des plus courantes est le recours aux produits des arbres des parcs agroforestiers (fruits, fleurs, graines, feuilles, écorce, racines) comme alternative de subsistance (Ouédraogo et al., 2013).

Les facteurs climatiques de dégradation des bas-fonds les plus remarqués par ces communautés sont les poches de sécheresse, la courte durée de la saison, les vents violents et les inondations. Les hommes alphabétisés perçoivent les vents violents comme principal cause de la dégradation des bas-fonds tandis que ceux qui ne sont pas alphabétisés indexent les inondations. Quant aux femmes alphabétisées, elles indiquent l'augmentation de la fréquence des séquences sèches comme principal facteur climatique de dégradation tandis que pour les femmes non alphabétisées, il s'agit de la diminution du nombre de jours pluvieux. Des travaux de recherche ont montré que le sexe et le niveau d'éducation influencent la perception du changement climatique ainsi que des options d'adaptation (Mertz et al., 2009 ; Sofoluwe et al., 2011 ; Loko et al., 2013). Ainsi, les femmes perçoivent- elles mieux les effets des facteurs climatiques tels que les poches de sécheresse et la réduction de la saison pluvieuse sur leurs activités agricoles. C'est certainement ce qui fonde leur perception de ces facteurs comme importants dans la dégradation des bas-fonds. Par ailleurs, les femmes sont plus touchées par le tarissement précoce des marres et des cours d'eau qui leur servent de points d'approvisionnement (Vissoh et al., 2012).

\section{Perception des indicateurs biophysiques de l'évolution des bas-fonds}

Les communautés du sous bassin versant du Nakanbé-Dem perçoivent la dégradation des bas-fonds à l'aide d'indicateurs physiques (élargissement de la zone centrale, placage sablo-gravillonnaire, érosion du sol, ravinement et asséchassent précoce des bas-fonds) et biologiques (diminution du couvert végétal et diminution $\mathrm{du}$ nombre d'arbres). Ces indicateurs biophysiques de dégradation des bas-fonds varient suivant le sexe et le niveau d'instruction. Plusieurs auteurs ont montré que les caractéristiques socio-économiques des communautés rurales telles que le sexe et le niveau d'instruction influencent la perception de la dégradation des bas-fonds (Jennifer et al., 2004 ; Sofoluwe et al., 2011). Le séjour moins long de l'eau dans les bas-fonds est bien perçu par les femmes parce qu'elles s'approvisionnent en eau à partir de ces zones humides (Vissoh et al., 2012). En plus de cet indicateur physique de dégradation des basfonds, les femmes perçoivent également la dégradation du sol et le ravinement des basfonds comme principaux indicateurs physiques 
de dégradation. Le niveau d'instruction n'a pas eu d'effet dans la perception des signes de dégradation des bas-fonds chez les femmes.

Les hommes non alphabétisés perçoivent la dégradation des bas-fonds à travers l'état du peuplement végétal, en l'occurrence la diminution du couvert végétal et $\mathrm{du}$ nombre d'arbres. La taille des ligneux ainsi que leur densité est un critère souvent utilisé pour évaluer la santé des bas-fonds (Mahamane et al., 2007; Ilboudo-Tapsoba et al., 2011). Quant aux hommes alphabétisés, les plaquages sablogravillonnaires ainsi que l'élargissement de la zone centrale des bas-fonds sont les principaux signes de dégradation de ces zones humides. En effet, dans la zone sahélienne, l'érosion par les eaux pluviales creuse par endroits des ravines et l'érosion régressive latérale opérée sur les berges des bas-fonds élargit leur lit et occasionne des dépôts de sable (Robert, 2011). Cet aspect est perçu par ces communautés, notamment les hommes alphabétisés.

\section{Perception de l'état des ressources phytobiologiques des bas-fonds dégradés}

$\mathrm{La}$ pression sur les bas-fonds occasionne un remaniement important des peuplements végétaux de ces zones humides. Cet indicateur est bien perçu par les communautés rurales qui reconnaissent certaines des espèces ayant disparu dans les bas-fonds ainsi que des espèces qui prolifèrent par suite de la dégradation de ces sites (Tableau 2).

Parmi les ligneux proliférant dans les bas-fonds dégradés, les paysans identifient couramment Balanites aegyptiaca (L.) Del; Acacia nilotica (L.) Willd. ex Del; Ziziphus mauritiana Lam; Combretum micranthum G. Don et Piliostigma reticulatum (DC.) Hochst. Lompo (2003) a également noté que ces espèces sont abondantes dans les agrosystèmes de la zone du sahel. Ces espèces identifiées sont dominées par des individus à plus grand degré de sclérophyllie (plantes dont les feuilles se transforment en épines afin de limiter les pertes d'eau dues à la sécheresse). Khoudia et al. (2014) ont montré que l'abondance de telles espèces dans un milieu s'explique par leur adaptabilité aux conditions pédoclimatiques dégradées.
Les espèces herbacées identifiées par les paysans comme proliférant dans les basfonds comprennent Digitaria horizontalis Willd, Pennisetum pedicellatum Trin., Hyptis spicigera Lam et Senna obtusifolia (L.) Irwin \& Barneby. Soulama (2016) a montré que la présence de ces espèces dans un milieu humide est liée à la dégradation de ce milieu par le pâturage. Outre le pâturage, plusieurs autres facteurs (coupe du bois, feux de brousse, etc.) concourent à l'installation de ces espèces ubiquistes dans ces milieux de bas-fonds. L'analyse de la flore identifiée par les paysans dans les bas-fonds dégradés corrobore plusieurs auteurs ayant caractérisé la flore des milieux dégradés (Mahamane et al., 2007 ; Khoudia et al., 2014; Soulama, 2016). En effet, les taxons cités sont dominés par les thérophytes (plantes annuelles à graines) et des espèces annuelles à distribution multirégionale (Pantropicale, Paléotropicale et Afrotropicale).

Quant aux espèces ligneuses en disparition dans les bas-fonds, les paysans identifient des espèces des milieux humides telles que le bouleau d'Afrique (Anogeissus leiocarpa), l'ébène d'Afrique (Diospyros mespiliformis) et Mitragyna inermis encore appelé Mitragyna africana. Ces espèces sont reconnues par plusieurs auteurs comme caractéristiques des milieux fréquemment inondés (Konaté, 2000; Mahamane et al., 2007; Habu et al., 2014). La modification des régimes hydriques serait un facteur ayant favorisé l'élimination de ces espèces qui jadis donnaient une tonalité humide à ces bas-fonds. Ainsi, les paysans perçoivent clairement l'érosion de la biodiversité végétale des écosystèmes des basfonds.

Les communautés du sous bassin versant du Nakanbé-Dem identifient les espèces telles que Andropogon pseudapricus Stapf, Andropogon tectorum Schumach. \& Thonn, Schizachyrium sanguineum (Retz.) Alston, comme ayant disparu des bas-fonds. L'analyse floristique des espèces identifiées par les paysans corrobore plusieurs auteurs qui ont montré que les espèces du genre Andropogon indiquent un milieu fertile, non dégradé (Mahamane et al., 2007 ; Soulama, 2016). Au contraire, la disparition de ces 
espèces des milieux de bas-fonds traduit une dégradation de ces sites.

Autant les paysans reconnaissent la dégradation des bas-fonds à travers l'identification de certaines espèces floristiques disparues, autant la bonne santé des écosystèmes des bas-fonds est reconnue par la prolifération d'autres espèces. La disparition de plusieurs espèces et l'apparition d'espèces ubiquistes peuvent permettre d'évaluer la dégradation des terres de bas-fonds. Ces connaissances endogènes des plantes indicatrices de dégradation peuvent servir dans le cadre de programme d'éducation environnementale des communautés.

\section{Conclusion}

Notre étude visait à étudier les indicateurs biophysiques de dégradation des bas-fonds des agriculteurs du sous bassin versant du Nakanbé Dem. Les résultats montrent que les paysans exploitant les basfonds ont des connaissances sur les facteurs de dégradation des bas-fonds et perçoivent les signes de dégradation ainsi que les espèces qui traduisent l'état de santé des bas-fonds. Le sexe et le niveau d'éducation des agriculteurs influencent leur perception des indicateurs de dégradation des bas-fonds. Ces indicateurs dépendent du sexe et du niveau d'éducation. Ces connaissances endogènes pourraient servir pour soutenir des programmes participatifs de restauration des bas-fonds dégradés.

\section{CONFLITS D'INTERETS}

Les auteurs déclarent n'avoir aucun conflit d'intérêts sur cet article.

\section{CONTRIBUTIONS DES AUTEURS}

$\mathrm{AI}$ et SS ont réalisé l'étude et participé au traitement des données. PZ et $\mathrm{EH}$ ont participé à la conception de projet de l'étude de recherche et supervisé le travail. Tous ces auteurs ont contribué à la rédaction du manuscrit soumis à votre journal pour publication.

\section{REMERCIEMENTS}

Les auteurs remercient l'Agence de l'Eau du Nakanbé (AEN) et le Secrétariat Permanent pour la Gestion Intégrée des
Ressources en Eau (SP/GIRE) qui ont financé l'étude.

\section{REFERENCES}

Bambara D, Bilgo A, Hien E, Masse D, Thiombiano A, HienV. 2013. Perceptions paysannes des changements climatiques et leurs conséquences socio environnementales à Tougou et Donsin, climats sahélien et sahélo-soudanien du Burkina Faso. Bulletin de la Recherche Agronomique du Bénin (BRAB), 16(74): 1840-7099. http://www.slire.net

CPCS. 1967. Commission de pédologie et de cartographie des sols de France. Réunion des 21, 22 et 23 septembre 1965 à EnsaGrignon, Paris, 12p. Ensa- Grignon, Paris, 12p.

Da D, Yacouba H, Yonkeu S . 2008. Unités morphopédologiques et gestion de la fertilité des sols dans le Centre-Nord du Burkina Faso par les populations locales. Int. J. Biol. Chem. Sci., 2(3): 306-315. DOI: 104314/ijbcsv2i339746

Diallo H, Bamba I, Barima YSS, Visser M, Ballo A, Vranken I, Maiga M, Bogaert J. 2011. Effets combinés du climat et des pressions anthropiques sur la dynamique évolutive de la végétation d'une zone protégée du Mali (Réserve de Fina, Boucle du Baoulé). Sécheresse, 22: 97107. DOI: $101684 / \mathrm{sec} 20110306$

Enete AA, Onyekuru AN. 2011. Challenges of Agricultural Adaptation to climate change: Empirical Evidence from Southeast Nigeria. Tropicultura, 29(4): 243-249. DOI: 101108/IJCCSM-082012-0045

Fontès J, Guinko S. 1995. Carte de végétation et de l'occupation d'un sol du Burkina Faso: notice explicative. Université de Ouagadougou. Ministère de la coopération française, Projet Campus, 67: 881-3101.

Habu R, Bakasso Y, Abdoulaye D, Maarouhi MI . 2014. Structure de la population de Boscia senegalensis ( Pers ) Lam Ex Poir suivant la toposéquense dans la commune de Simiri (Niger). Journal of Animal and Plant Sciences, 23(3), 3657-3669. DOI: http://wwwmelewaorg/JAPS

Hounkpetin C. 2003: Contribution à la mise en 
valeur du bas-fond de Okeita dans la commune de Pobe (Departement du Plateau). Thèse d'ingénieur agronome, FSA-UAC ,134p.

Huat J, Fusillier J, Dossou-yovo E, Lidon B, Kouyaté AM, Touré A, Simpara MB, Hamadoun A. 2019. Benefits and limits of inland valley development to enhance agricultural growth: a farmers , perception approach in southern Mali. Environment, Development and Sustainability. DOI: https://doi.org/10.1007/s10668-01900466-6

Ilboudo-Tapsoba E, Tankoano H, Ouédraogo M, Dicko IO, Sanon A .2011. Diversité des insectes actifs au sol dans quatre écosystèmes de bas-fonds du Burkina Faso : importance pour la détermination de bio-indicateurs caractérisant ces milieux. Int. J. Biol. Chem. Sci., 5(2): 724-738. DOI: 104314/ijbcsv5i272146

INSD. 2009. Projections démographiques de 2007 à 2020 par région et province. Ouagadougou.

Jennifer SB, Ann EB, William GA. 2004. Neighborhood Social Change and Perceptions of Environmental Degradation. Population and Environment, $\quad \mathbf{2 5}(2)$ : $\quad 31-39$ DOI: 10.1023/B:POEN.0000015559.5381

Khoudia N, Ndiaye O, Diallo A, Guisse, A .2014. Flore et structure de la végétation ligneuse le long de la Grande Muraille Verte au Ferlo, nord Sénégal. Journal of Applied Biosciences, 79: 6938-6946. DOI: http://dx.doi.org/10.4314/jab.v79i1.15

Konaté L. 2000. Les bas-fonds dans le réseau hydrographique du fleuve Gambie. Mémoire de Thèse de l'Univsersité Cheikh Anta Diop de Dakar. Département de Géographie. 207p.

Lebrun JP, Stork A. 1991. Enumération des Plantes à Fleurs d'Afrique Tropicalevolume 1; Généralités et Annonaceae à Euphorbiaceae et Pandaceae. Conservatoire et Jardin Botaniques : Genève ; $249 \mathrm{p}$.

Loko YL, Dansi A, Agre a P, Akpa N, DossouAminon I, Assogba P, Dansi M, Akpagana K, Sanni A. 2013. Perceptions paysannes et impacts des changements climatiques sur la production et la diversité variétale de l'igname dans la zone aride du nord-ouest du Bénin. Int. J. Biol. Chem. Sci., 7(2): 672-695. DOI: 10.4314/ijbcs.v7i2.23

Lompo O. 2003. Les stratégies paysannes de lutte contre la dégradation des terres dans le Sahel Burkinabé. Mémoire de Maîtrise, Université de Ouagadougou, 134p.

Mahamane A, Mahamane S, Yacouba B, Issaka A, Ichaou A, Saley K. 2007. Analyse diachronique de l'occupation des terres et caractéristiques de la végétation dans la commune de Gabi (région de Maradi, Niger). Sécheresse, 18(4): 296304. DOI: $10.1684 / \mathrm{sec} .2007 .0105$

Malam AM. 2007. Approche méthodologique pour la constitution d' une base de données pour la surveillance des systèmes hydrogéomorphologiques du bassin de Kori Dantiandou (Degré carré de Niamey ). Mémoire de DEA, Université Abdou Moumouni de Niamey (UAM), Niger. $115 \mathrm{p}$.

Mertz O, Mbow C, Reenberg A, Awa D. 2009. Farmers ' Perceptions of Climate Change and Agricultural Adaptation Strategies in Rural Sahel. Environmental Management, 43: 804-816 : DOI 10.1007/s00267-008-9197-0

Nacoulma-Ouédraogo OG. 1996. Plantes médicinales et pratiques médicales traditionnelles au Burkina Faso: Cas de plateau central. Tome 1,380p.

Nana TJ, Thiombiano T. 2018. Adoption of Adaptation Strategies for Climate Change: Case of Burkina Faso Farmers. Journal of Agriculture and Environmental Sciences, 6(1): 53-65. DOI: https://doi.org/10.15640/jaes.v7n1a6

Oloukoi J, Mama VJ, Fulbert E, Agbo B. 2006. Modélisation de la dynamique d'occupation des terres dans le département des collines au Bénin. Télédétection, 6(4): 305-323. https://www.researchgate.net/publication 1279851907

Ouédraogo M, Ouédraogo $\mathrm{D}$, Thiombiano T, Hien M, Lykke AM. 2013. Dépendance économique aux produits forestiers non 
ligneux : cas des ménages riverains des forêts de Boulon et de Koflandé au SudOuest du Burkina Faso. Journal of Agriculture and Environment for International Development, 107(1): 4572. DOI: http://dx.doi.org/10.12895/jaeid.20131.9 8

Raunet M. 1985. Bas-fonds et riziculture en Afrique: Approche structurale comparative. L'Agronomie Tropicale, 40: 181-201. DOI: http://agroecologie.cirad.fr

Robert E. 2011. Les risques de pertes en terre et en eau dans le bassin versant de la Doubégué (Burkina Faso): pour une gestion intégrée. Thèse de doctorat de l'Université Michel de Montaigne Bordeaux. 541p. https://hal.archivesouvertes.fr/tel-01090397

Sambare O, Ouedraogo O, Wittig R, Thiombiano A. 2011. Diversité et écologie des groupements ligneux des formations ripicoles du Burkina Faso (Afrique de l'Ouest). Int. J. Biol. Chem. Sci., 4(5): 1782-1800. DOI: 10.4314/ijbcs.v4i5.65587

Sofoluwe NA, Tijani AA, Baruwa OI. 2011. Farmers 'perception and adaptation to climate change in Osun State, Nigeria. African Journal of Agricultural, 6(20): 4789-4794. DOI: 10.5897/AJAR10.935

Souberou K, Agbossou KE, Ogouwale E. 2017. Inventaire et caractérisation des bas-fonds dans le bassin versant de l'Oti au Bénin à l'aide des images Landsat et ASTER DEM. International Journal of Environment, Agriculture and Biotechnology (IJEAB), 2(4):1601-1623. http://dx.doi.org/10.22161/ijeab/2.4.20

Souberou KT, Barre IO, Yabi I, Ogouwale E. 2018. Fondements géographiques de la valorisation agricole des bas-fonds au
Sud du bassin versant de l'Oti (Bénin). European Scientific Journal, ESJ, 14(21): 1601-1623.

DOI: 10.19044/esj.2018.v14n21p136

Soulama S. 2016. Types d'utilisation des terres et incateurs de dégradation de la biodiversité dans les réserves de Pama et les agrosystèmes ( Sud-est du Burkina Faso). Mémoire de thèse de l'Université Ouaga I Pr Joseph KI - ZERBO. 199p.

Soulama S, Nacoulma B, SavadogoP atrice S, Bachmann Y. 2015. Do floristic composition, plant species abundance and vegetation structure in Sudanian wetlands vary according to conservation status? Global Journal of Wood Science, Forestry and Wildlifel, 3(3): 83-93. DOI: http://www.globalscienceresearchjournal s.org/

Thiombiano A, Schmidt M, Dressler S, Ouédraogo A, Hahn K, Zizka G. 2012. Catalogue des plantes vasculaires du Burkina Faso. Boissiera. Mémoires de botanique systématique, 391p.

Vissoh PV, Tossou RC, Dedehouanou H, Hervé, G, Olivier, CC, Simplice, D V, Euloge KA . 2012. Perceptions et stratégies d'adaptation aux changements climatiques: le cas des communes d 'Adjohoun et de Dangbo au Sud-Est Bénin. Les Cahiers d'Outre-Mer Revue de Géographie de Bordeaux, 260(65): 479-492. DOI: 10.4000/com.6700

Yacouba H, Da DEC, Yonkeu S, Zombre P, Soule M . 2002. Caractérisation du ruissellement et de l'érosion hydrique dans le bassin supérieur du Nakambé (Burkina Faso). In: 5ème Conférence Interrégionale sur l'Environnement et l'Eau. Envirowater pp610-615. https://wwwresearchgatenet/publication/ 286078785 